\title{
The McDaniel family: The impact of small-group clinical teaching early in the medical school curriculum
}

DANIEL J. RAUB, D.O., M.A

HELEN H. BAKER, PH.D

MARY PAT MANN, ED.S.

Athens, Ohio

\begin{abstract}
The implementation of Medical Care for the McDaniel Family early in our four-year, systems-oriented curriculum is discussed. The instructional package includes six video vignettes depicting delivery of family medical care in the ambulatory setting, corresponding student Study Guide, an Instructor's Manual, and a problemoriented record system. We sought to provide vicarious clinical experience, influence more students to enter family practice, teach mastery in writing progress notes, and promote smallgroup teaching. Our research thrust was course evaluation, and was focused on changes in attitudes. Statistically significant results of data analysis suggest program success, but are surprising.
\end{abstract}

Part of the legislative mandate for the Ohio University College of Osteopathic Medicine (OU-COM) is to graduate physicians who will enter into primary care practice in medically underserved areas, and we are constantly seeking curricular innovations to accomplish this goal. We have seen benefits from early contact between family medicine faculty and medical students.

At the 1983 Society of Teachers of Family Medicine annual spring conference in Boston, several of our staff heard a presentation on small-group methods for introducing family medicine at the Medical College of Georgia. A similar program, Medical care for the McDaniel family, ${ }^{1}$ had already been packaged by the Duke-Watts Family Medicine Program, Duke University Medical Center, Durham, North Carolina. Later that year, we decided to add this module to the history and physical component of the second Osteopathic Clinical Practice (OCP) course. OCP II is presented in the second quarter of the first year, during the predominately basic science portion of our systems-oriented curriculum.

The OCP sequence in our curriculum spans six quarters (two years). It includes history taking and physical examination, osteopathic principles and practice, psychosocial skills, geriatrics, substance abuse, ethics, professional issues, and other clinically oriented components. The OCP courses comprise the only formal clinical exposure students receive in the first year, although some students make personal arrangements for clinical experience during times off. The second-year curriculum includes progressively more clinical material, in addition to continuation of the OCP sequence.

\section{Instructional package}

The McDaniel family package consists of six videotaped vignettes, each lasting 8-10 minutes, which depict simulated family practice encounters with Johnnie and Susie McDaniel, their parents Jack and Jane, and Jack's mother (Grandma Beulah). The practice association includes physicians Harold Williams and Mary Smith and physician assistant Bruce Morris.

A student study guide is provided for each vignette. A set of blank medical records, patterned after the Problem-Oriented Medical Record (POMR) developed by Weed, tested by Cross and Bjorn, and advocated by Hurst and others, ${ }^{1}$ is supplied for each McDaniel family member. The forms allow students to record the three major POMR elements, including problem list (and medication history), database (demographics, history, immunizations, diagnostic results, and so forth), and progress notes. History sheets differ according to patient age (adult or child) and gender.

An instructor's manual is also provided. It discusses the program, assists group leaders in class preparation, and includes sample test questions and a "student perception evaluation," which will be described later.

Suggested instructional tasks include showing the videotapes, construction and maintenance of 
medical records, identification and discussion of individual and family problems, instructor presentation of mini-lectures related to topics and issues raised, and student study after class. The entire educational module offers a method to expose preclinical medical students to aspects of family medicine practice in the ambulatory care setting. The authors recommend presentation to groups of 15-20 students near the end of the basic science curriculum.

\section{Rationale and objectives}

To emphasize the importance of family medicine at our institution, and to offer vicarious clinical exposure as early as possible in the curriculum, we introduced the McDaniel family program about a year before its design would intend. We adapted it by giving less attention to advanced medical content and concentrating on general concepts, particularly the psychosocial, pragmatic, and experiential aspects of family practice. We particularly emphasized students' abilities to write problemoriented progress notes in proper SOAP (subjective, objective, assessment, plan) format, ${ }^{1}$ using correct English language (accuracy of medical content not critical). Along with an associated lecture, the McDaniel family now serves as the students' introduction to charting and medical records.

Specific course objectives pertinent to the McDaniel family, as conveyed to the students in the course syllabus, are as follows:

(1) Record historic and physical findings to the standards set by the course content.

(2) Define and describe family dynamics, and give examples.

(3) Identify and describe features that make the role of the family practitioner unique and distinctive in the provision of health care.

(4) Define the team approach to health care, demonstrate the ability to interact effectively within a medical group, and identify factors personally discovered to impact upon group dynamics.

(5) State risk factors for coronary heart disease, discuss preventive medicine, and categorize and give examples of other risk factors.

(6) Write a concise progress note in proper SOAP format (content not critical).

We hoped to increase the likelihood that students would ultimately enter family practice or other areas involving primary patient care, including general internal medicine, pediatrics, emergency medicine, and perhaps obstetrics/gynecology. We expected that use of small-group teaching methods would yield immediate as well as future educational benefits for the students.

\section{Planning summary}

General policies, objectives, and procedures were developed after many meetings with participating physicians and Educational Development and Resources staff. A training session in small-group dynamics and related teaching methods was conducted during one of the organizational meetings for involved faculty. The six vignettes also were previewed and discussed during this meeting. Multiple memos before, during, and after the course helped to maximize consistency of presentations across groups and from year to year.

At the same time, group autonomy and individual group needs were not only recognized but acknowledged as desirable and necessary. Instructors were encouraged to capitalize on and pursue appropriate opportunities to cultivate individuality. Within broadly described guidelines, little restraint was placed upon the exercise of effective teaching prerogatives tailored to particular groups or leaders.

\section{Course implementation}

First-year students meet in eight small groups of 12-14 students each, the same "learning team" groups used in the osteopathic principles and practice laboratory component of OCP courses. These groups are constituted before school starts, by an alphabetic process using students' last names. Four groups are formed, using every fourth last name; these groups are then divided in half. The process essentially is random, and the student groups generally are maintained the same for purposes of laboratory assignments throughout the curriculum. Occasionally, a student may be reassigned to another group because of scheduling necessities.

Each group works with a full-time academic physician actively engaged in a practice of family medicine. Physicians' practices variably include general adult medicine, obstetrics, pediatrics, geriatrics, and/or preventive medicine, and either predominantly ambulatory or mixed outpatient and inpatient practice. With one exception, all participating physicians currently are certified by the American Osteopathic Board of General Practice; one is also a diplomate of the American Board of Family Practice.

The six videotape vignettes are shown successively in weeks $2,3,4,6,7$, and 8 of the 10 -week quarter, at the beginning of sessions lasting 1-2 hours. Students receive their study guides before the videotapes are shown, rather than after as recommended. This helps to compensate for the students' early point in clinical training and allows them to review materials before class if desired. 


\section{non-insulin-dependent}

\section{Diabetes and hypertension}

Because non-insulin-dependent diabetes mellitus is frequently accompanied by hypertension, it is important to select an oral hypoglycemic agent that rarely causes water retention. In contrast to chlorpropamide, MicRONASE, when administered alone, may produce a mild diuresis. ${ }^{1}$

All sulfonylureas, including MicRoNASE, can cause severe hypoglycemia. Proper patient selection, dosage, and instructions are important.

No other oral antidiabetic agent fits the realities of life better than

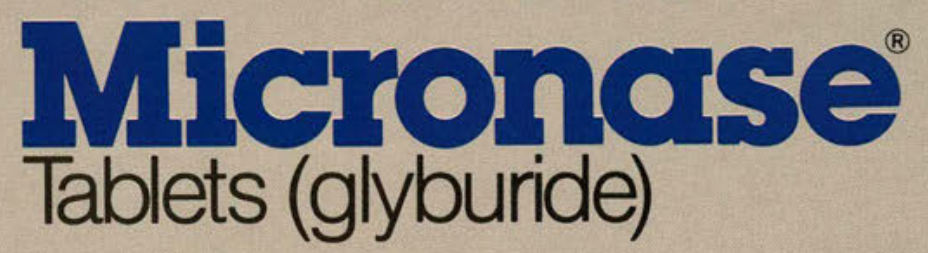

Usual starting dosage $-2.5 \mathrm{mg}$ to $5 \mathrm{mg}$ once a day

When diet alone fails...MicronASE

Please see adjacent page for brief summary of prescribing information.

'Moses AM, Howanitz J, Miller M: Diuretic action of three sulfonylurea drugs. Ann Intern Med 1973;78:541-544. 

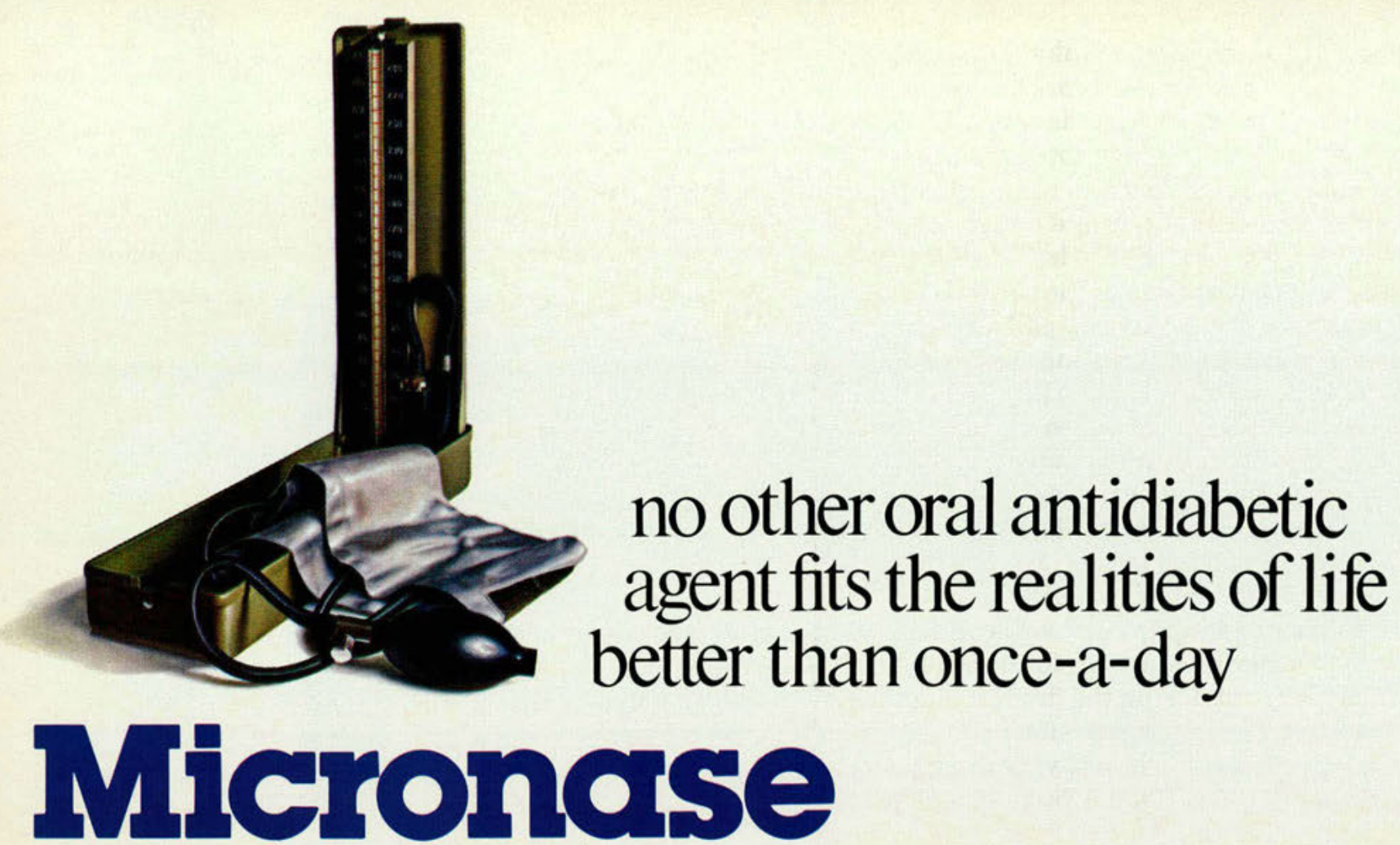

Some patients, particularly those receiving more than 10 mg daily, may have a more satisfactory response with twice-a-day dosage.

\section{Micronase Tablets (glyburide)}

CONTRAINDICATIONS: MICRONASE Tablets are contraindicated in patients with: 1. Known hypersensitivity or allergy to the drug. 2. Diabetic ketoacidosis, with or without coma. This condition should be treated with insulin. 3. Type I diabetes mellitus, as sole therapy.

SPECIAL WARNING ON INCREASED RISK OF CARDIOVASCULAR MORTALITY: The administration of ora hypoglycemic drugs has been reported to be associated with increased cardiovascular mortality as compared to treatment with diet alone or diet plus insulin. This warning is based on the study conducted by the University Group Diabetes Program (UGDP), a long-term prospective clinical trial designed to evaluate the effectiveness of glucose-lowering drugs in preventing or delaying vascular complications in patients patients with noninsulin-dependent diabetes. The study involved 823 patients who were randomly assigned to one of four treatment groups (Diabetes, 19 [Suppl 2]:747-830, 1970).

UGDP reported that patients treated for 5 to 8 years with diet plus a fixed dose of tolbutamide (1.5 grams per day) had a rate of cardiovascular mortality approximately $2 \frac{1}{2}$ times that of patients treated with diet alone. A significant increase in total mortality was not observed, but the use of tolbutamide was discontinued based on the increase in cardiovascular mortality, thus limiting the opportunity for the study to show an increase in overall mortality Despite controversy regarding the interpretation of these results, the findings of the UGDP study provide an adequate basis for this warning. The patient should be informed of the of the UGDP study provide an adequate basis for this warning. The patient should

Although only one drug in the sulfonylurea class (tolbutamide) was included in this study, it is prudent from a safety standpoint to consider that this warning may apply to other oral hypoglycemic drugs in this class. in view of their close similarities in mode of action and chemical structure.

PRECAUTIONS: General-Hypoglycemia: All sulfonylureas are capable of producing severe hypoglycemia Proper patient selection and dosage and instructions are important to avoid hypoglycemic episodes. Renal or hepatic insufficiency may increase the risk of serious hypoglycemic reactions. Elderly, debilitated or malnourished patients, and those with adrenal or pituitary insufficiency, are particularly susceptible to the hypoglycemic action of glucose-lowering drugs. Hypoglycemia may be difficult to recognize in the elderly and in people who are taking beta-adrenergic blocking drugs. Hypoglycemia is more likely to occur when caloric intake is deficient, after severe or prolonged exercise, when alcohol is ingested, or when more than one glucose lowering drug is used. Loss of Control of Blood Glucose' In diabetic patients exposed to stress such as fever, trauma intection or surgery, a loss of control may occur. It may then be necessary to discontinu MICRONASE and administer be assessed before classifying a patient as a secondary failure. Information for Patients: Patients should be be assessed before classifying a patient as a secondary failure. Information for Patients: Patients should be
informed of the potential risks and advantages of MICRONASE and of alternative modes of therapy. They also should be informed about the importance of adherence to dietary instructions, of a regular exercise program, and of regular testing of urine and/or blood glucose. The risks of hypoglycemia, its symptoms and treatment, and conditions that predispose to its development should be explained to patients and responsible family members. Primary and secondary failure should also be explained Laboratory Tests: Response to Measurement of glycosylated hemoglobin levels may be helpful in some patients. Drug Interactions: Th hypoglycemic action of sulfonylureas may be potentiated by certain drugs including nonsteroidal antiinflammatory agents and other drugs that are highly protein bound, salicylates, sulfonamides, chloramphenicol, probenecid, coumarins, monoamine oxidase inhibitors, and beta adrenergic blocking agents. Certain drugs tend to produce hyperglycemia and may lead to loss of control. These drugs include the thiazides and other diuretics, corticosteroids, phenothiazines, thyroid products, estrogens, oral contraceptives, phenytoin, nicotinic acid sympathomimetics, calcium channel blocking drugs, and isoniazid A potential interaction between oralmiconazole and oral hypoglycemic agents leading Carcinogenesis, Mutagenesis, and Impairment of Fertility: Studies in rats at doses up to $300 \mathrm{mg} / \mathrm{kg} / \mathrm{day}$ for 18 months showed no carcinogenic effects. Glyburide is nonmutagenic when studied in the Salmonella microsome test (Ames test) and in the DNA damage/alkaline elution assay. Pregnancy: Teratogenic Effects: Pregnancy Category B. Reproduction studies in rats and rabbits have revealed no evidence of impaired fertility or harm to the fetus due to glyburide. There are no adequate and well controlled studies in pregnant women. This drug should be used during pregnancy only if clearly needed. Insulin should be used during pregnancy to maintain blood glucose as close to normal as possible. Nonteratogenic Effects: Prolonged prevere hypolycemia (4 to 10 lays) has been reported in as possible. Nonteratogenic ellects. Prolonged sulfonylurea drug at the time of delivery. MICRONASE should be discontinued at least two weeks before the sulfonylurea drug at the time of delivery. MICRONASE should be discontinued at least two weeks before the
expected delivery date. Nursing Mothers: Some sulfonylurea drugs are known to be excreted in human milk. Insulin therapy should be considered. Pediatric Use: Safety and effectiveness in children have not been established.

ADVERSE REACTIONS: Hypoglycemia: See Precautions and Overdosage sections. Gastrointestinal Reactions: Cholestatic jaundice may occur rarely: MICRONASE Tablets should be discontinued if this occurs. Gastrointestinal disturbances, eg nausea, epigastric fullness, and heartburn, are the most common reactions, having occurred in $1.8 \%$ of treated patients during clinical trials. They tend to be dose-related and reactions, having occurred in $18 \%$ of treated patients during clinical trials. They tend to be dose-related and
may disappear when dosage is reduced. Liver function abnormalities, including isolated transaminase elevations, have been reported. Dermatologic Reactions: Allergic skin reactions, e.g. pruritus, erythema, urticaria, and morbilliform or maculopapular eruptions, occurred in $1.5 \%$ of treated patients during clinical trials. These may be transient and may disappear despite continued use of MICRONASE; if skin reactions persist, the drug should be discontinued. Porphyria cutanea tarda and photosensitivity reactions have been reported with sulfonylureas. Hematologic Reactions: Leukopenia, agranulocytosis, thrombocytopenia, hemolytic anemia, aplastic anemia, and pancytopenia have been reported with sulfonylureas. Metabolic Reactions: Hepatic porphyria and disulfiram-like reactions have been reported with sulfonylureas: however. Reactions: Hepatic porphyria and disulfiram-like reactions have been reported with sulfonylureas; however,
hepatic porphyria has not been reported with MICRONASE and disulfiram-like reactions have been reported very rarely. Cases of hyponatremia have been reported with glyburide and all other sulfonylureas, most often in patients who are on other medications or have medical conditions known to cause hyponatremia or increase release of antidiuretic hormone. (SIADH) secretion has been reported with certain other sulfonylureas, and it has been suggested that these sulfonylureas may augment the peripheral (antidiuretic) action of ADH and /or increase release of $\mathrm{ADH}$.

OVERDOSAGE: Overdosage of sulfonylureas, including MICRONASE Tablets, can produce hypoglycemia. If hypoglycemic coma is diagnosed or suspected, the patient should be given a rapid intravenous injection of concentrated ( $50 \%$ ) glucose solution. This should be followed by a continuous infusion of a more dilute (10\%) glucose solution at a rate which will maintain the blood glucose at a level above $100 \mathrm{mg} / \mathrm{dL}$. Patients should be closely monitored for a minimum of 24 to 48 hours, since hypoglycemia may recur after apparent clinical recovery.

Caution: Federal law prohibits dispensing without prescription. Store at controlled room temperature $15^{\circ}-30^{\circ} \mathrm{C}\left(59^{\circ}-86^{\circ} \mathrm{F}\right)$. Dispensed in well closed containers with safety closures. Keep container tightly closed For additional product information see your Upjohn representative.

\section{Upjohn}

THE UPJOHN COMPANY, Kalamazoo, MI 49001, USA 
Students are encouraged to take notes while viewing the videotapes, for use in preparing their problem-oriented medical records and SOAP notes. Patient charts and progress notes usually are completed out of class, before the next session, rather than using class time for these chores.

The physicians act as moderators to facilitate discussion and expansion of the positive and negative aspects of the simulated patient encounters. They also give mini-lectures and inject comment on personal experiences related to the topics. They are encouraged to review the video vignettes, refer to the respective student study guides, and study the instructor's manual prior to class.

As course coordinator, Dr. Raub frequently visited and observed other groups, helping to ensure a measure of group consistency, checking that necessary supplies and equipment were on hand, and serving as a resource person for questions. (This was more frequent during the first year, but less so in subsequent years.) Educational Development and Resources staff occasionally sat in with a group to observe the educational process and to provide constructive feedback.

\section{Student grading}

Students are required to participate in discussion to receive a satisfactory grade for each session. Each student receives a grade of satisfactory, marginal, or unsatisfactory for each session. (To date, no student has received a rating less than satisfactory for any session from his or her instructor.) Students "peer-review" a classmate's SOAP notes after each class and before the next session. Questions related to previous session SOAP notes are entertained at the start of the next session. Instructors review and "trial grade" the peer-review notes from session III between weeks 4 and 6 of the quarter, to be certain that the peer-review process is working as intended, and that mistakes are not being repeated. Finally, instructors actually grade the notes from session VI after week 8 .

Notes for problems presented in the last McDaniel family encounter (session VI) must receive passing grades (satisfactory or marginal) for students to pass the overall OCP course. Students can rewrite notes up to three times if necessary to pass. Suggested standards for instructor grading are as follows: For format, 0 errors = satisfactory, 1 error $=$ marginal, and 2 or more errors $=$ unsatisfactory; for language, 1 error = satisfactory, 2 errors $=$ marginal, and 3 or more errors $=$ unsatisfactory.

The achievement of mastery in writing medical progress notes was (and remains) a major objective of the McDaniel family program in our curriculum.
Only rarely has a student had to rewrite notes to meet course requirements, and no student has yet failed because of the McDaniel family component.

\section{Implementation summary}

The McDaniel family program has now been presented during the winter quarter for three consecutive years $(1984,1985$, and 1986). We have maintained its content and format essentially unchanged. Instructors remained the same each year, with minor exceptions: One faculty member left the college after the first year, one was added for the second and third years, one dropped out the second year only, and two shared responsibilities for a group of students the third year because of scheduling conflicts for clinical responsibilities. (After further evaluation, this latter modification may provide some new options for handling small groups.) In addition, two predoctoral family medicine fellows assisted with instruction and facilitation in some groups the third year.

Initially, we also hoped that Medical care for the McDaniel family could replace the past personal history, family history, and social history segments of our history taking and physical examination curricular component. This expectation proved to be unreasonable after the first year's experience. We found that time constraints of the program as packaged (and delivered) prevented inclusion of so much additional material. Subsequently, we reintroduced these segments into OCP II (the same quarter in which the McDaniel family component was added), but with more of a preventive medicine orientation.

\section{Program evaluation}

Three approaches were used to evaluate the impact of Medical care for the McDaniel family as implemented in the curriculum at OU-COM: (1) results of a student attitude survey included in the instructor's manual; (2) results of two instruments we developed to elicit student feedback on instruction; and (3) feedback during and after the course from involved faculty and Educational Development and Resources staff regarding effectiveness of the program.

\section{Attitude survey}

A "student perception evaluation" questionnaire (called the "attitude survey" in this paper) is included as Appendix B in the McDaniel family instructor's manual. The survey contains 17 items (listed in Table 1), which students rated on a 5-point scale, with 5 = "strongly agree" and 1 = "strongly disagree." Items are stated both positively and negatively. For ease of interpretation, we recoded 
the negative items in analysis to make all items positive.

The original survey form was designed for one use at the end of the course; it asked students to indicate their current attitudes and to recall their attitudes before the course. We altered this process by using multiple administrations of the survey. We assured student anonymity (and hopefully honest response) while still maintaining tracking abilities by asking students to identify themselves "generically," by the first name of their best friend in junior or senior high school and the make/year/ color of their first automobile. (In retrospect, this method of tracking proved to be cumbersome. In the future, we plan to use more specific and stable identifiers, such as mother's birthdate.)

Except for the first year, the attitude survey was administered to students three times: (1) at the time of medical school entry (orientation day, or "before school"); (2) before the course; and (3) after the course. (Our initial decision to implement this new component was too late the first year to allow for administration on orientation day.) With each administration of the survey, we asked students to use the same anonymous identifiers, so that we could track responses. We obtained usable, paired responses for 85-95 percent of the students each year (of approximately 100 students per class).

Administration of the survey "before school" was done to control for changes in attitude that could be attributed to events occurring between start of school and start of the course, such as students interacting with physicians in the learning teams mentioned earlier. Physicians who serve as moderators for the McDaniel family program are predominantly the same individuals who serve as osteopathic principles and practice learning team leaders throughout the OCP curriculum. We try to keep groups and instructors intact during the first year, although some administrative variations occur.

According to the original author, the Student Perception Evaluation was designed to include 13 scales of one or two items each. ${ }^{2}$ Each scale was intended to measure a specific student attitude toward family medicine, such as the need for family medicine, or the value of health teams. To validate these structures, we used principal factors analysis with varimax rotation to examine the underlying structure of the survey. Each year was analyzed separately to determine whether the factor structure was stable from year to year. For each analysis, we included all forms returned for that year.

Each analysis revealed three factors that could be retained for rotation, and the same three factors were found for each year. Factors were retained
TABLE 1. ITEMS ON THE STUDENT PERCEPTION EVALUATION.

(1) It is important to consider patients as individuals.

( 2) It is important to consider patients as members of a family unit.

( 3) Psychosocial problems are not as important as purely medical problems for the physician to treat.

( 4) Psychosocial problems can be easily distinguished from purely medical problems.

( 5) Knowledge of family interactions can be important when managing individual patients.

( 6) The doctor-patient relationship has little bearing on patient compliance and satisfaction.

( 7) There is little need for family medicine as a medical specialty.

( 8) Family physicians today are no more academically acceptable than were the general practitioners of twenty years ago.

(9) Family medicine is a possible career choice for me.

(10) Some form of primary care medicine is a possible career choice for me.

(11) Many functions formerly performed entirely by physicians can be accomplished now equally well by midlevel practitioners such as physicians assistants and nurse practitioners.

(12) The team approach utilizing personnel from multiple disciplines does little to enhance health care delivery when compared with the care delivered by physicians alone.

(13) Good medicine can be practiced in the ambulatory setting, often without hospitalizing the patient.

(14) Hospitalization itself can be detrimental to a patient's health.

(15) The cost of medical care is often as important a consideration as the choice of medications in patient management.

(16) The individual physician can do little to affect costcontainment in health services.

(17) Learning in medical school is facilitated by interacting with faculty on as near a peer level as possible.

based on the scree test and on interpretability of factors. ${ }^{3}$ Table 2 presents the factor loadings, eigenvalues, and percent of variance explained for each year of the analysis. Based on the factor analyses, we constructed three scales-psychosocial attitude, ambulatory medicine attitude, and career choice. Table 3 lists the items associated with each scale. One item (no. 17, peer interaction with faculty) was eliminated from further analysis because of low factor loadings.

Scores were computed for each questionnaire on each scale by calculating the average of the item responses. The three scale scores provided data for all subsequent analysis. Chronbach's alpha reliability coefficients were computed for each scale for each year. These reliability coefficients ranged from 0.59 to 0.81 . The results of the factor analyses and the scale reliabilities indicate that the Student Perception Evaluation measures three components of student attitudes toward family medicine. These components remained stable across three classes of incoming medical students, thus providing reliable measures of student attitudes. 


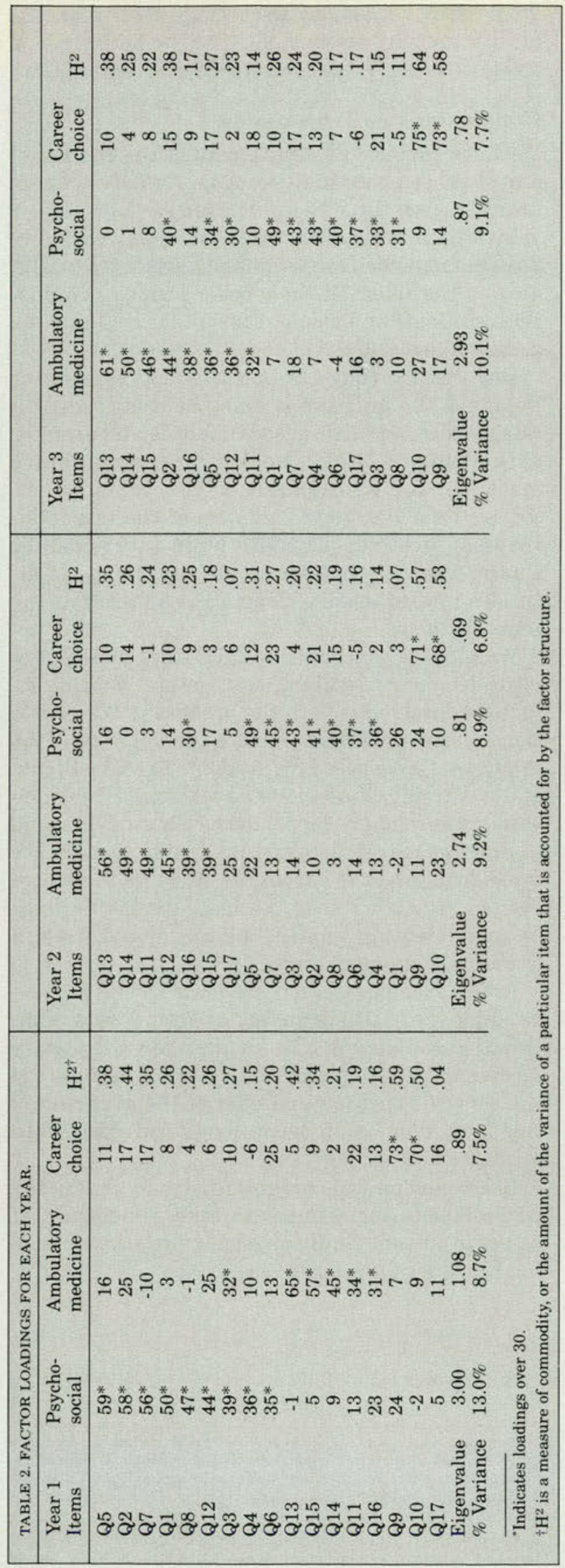

TABLE 3. ATTITUDE SCALES WITH RELIABILITY COEFFICIENTS.

\section{Psychosocial attitude scale}

(1) It is important to consider patients as individuals.

( 2) It is important to consider patients as members of a family unit.

( 3) Psychosocial problems are not as important as purely medical problems for the physician to treat.

( 4) Psychosocial problems can be easily distinguished from purely medical problems.

( 5) Knowledge of family interactions can be important when managing individual patients.

(6) The doctor-patient relationship has little bearing on patient compliance and satisfaction.

( 7 ) There is little need for family medicine as a medical specialty.

( 8) Family physicians today are no more academically acceptable than were the general practitioners of twenty years ago.

Cronbach's alpha: Year 1-.70 Year 2-.59 Year 3-.62

Ambulatory medicine attitude scale

(11) Many functions formerly performed entirely by physicians can be accomplished now equally well by midlevel practitioners such as physicians assistants and nurse practitioners.

(12) The team approach utilizing personnel from multiple disciplines does little to enhance health care delivery when compared with the care delivered by physicians alone.

(13) Good medicine can be practiced in the ambulatory setting, often without hospitalizing the patient.

(14) Hospitalization itself can be detrimental to a patient's health.

(15) The cost of medical care is often as important a consideration as the choice of medications in patient management.

(16) The individual physician can do little to affect costcontainment in health services.

Cronbach's alpha: Year 1-.61 Year 2-.64 Year 3-.61

Career choice attitude scale

( 9) Family medicine is a possible career choice for me.

(10) Some form of primary care medicine is a possible career choice for me.

Cronbach's alpha: Year 1-.74 Year 2-.77 Year 3-.81

For each year, we computed repeated-measures multivariate analysis of variance (MANOVA) for each scale. Two independent factors were examined-the small group to which the student belonged, and the time of the survey administration. The design used calculated, simple, one-way ANOVAs to assess small group effects before applying multivariate tests for the repeated measures and interaction effects. ${ }^{4}$ Alpha was set at .01 .

For each of the three years, significant gains were obtained on the time of administration factor on the ambulatory medicine attitude scale: Students felt significantly more positive toward ambulatory medicine after the program. Table 4 summarizes the MANOVA test criteria for this scale for each year. The psychosocial attitude and career choice scales showed positive trends, but changes were not statistically significant.

Because basic science courses comprise the rest of 
TABLE 4. MANOVA CRITERIA FOR THE HYPOTHESIS OF NO TIME EFFECT AMBULATORY MEDICINE ATTITUDE SCALE.

\begin{tabular}{lll} 
Year 1 & Year 2 & Year 3 \\
\hline $\begin{array}{c}\text { Wilk's criterion } \\
(\mathrm{L})=0.916\end{array}$ & Wilk's criterion & Wilk's criterion \\
$\mathrm{F}(1,76=6.97$ & $\mathrm{F}(2,62)=0.675$ & $(\mathrm{~L})=0.728$ \\
Prob $>\mathrm{F}=0.0100$ & Prob $>\mathrm{F}=0.0001$ & $\mathrm{~F}(2,78)=14.58$ \\
\end{tabular}

the first-year curriculum, we attribute the positive change in attitudes to the OCP course, of which the McDaniel family program was a major component.

\section{Student feedback on instruction}

Students evaluated the McDaniel family component as part of our standard course evaluation procedures each year. Student ratings included responses on a standardized form and a special questionnaire that focused on the McDaniel family program. Response rates ranged from 60 percent (1986) to 95 percent(1984). Each year, OCP II received high ratings compared to other secondquarter courses, and also compared to OCPI and III in the first year. In 1986 , students rated the McDaniel family as third of seven components in the OCP II course.

Ratings of teaching by individual family medicine faculty ranged from 3.73 to 5.00 (on a 5-point scale), with a median rating of 4.75 . In every case, student ratings of the faculty member's teaching in the McDaniel family component were substantially higher than ratings of his/her other teaching activities (lecture or clinical laboratories).

Each year, 70 to 100 percent of the students returning the special questionnaire believed that they had achieved all of the stated objectives of the program. The most popular aspects of the program were: (1) its relevance to medical practice ("...the most medically pertinent exercise we've done yet," “...made family medicine seem important"); (2) the chance for discussion ("We learned from the experiences of the other students," "...the small group setting...made it easier to talk and interact"); and (3) learning to write progress notes. The least popular aspects of the program were: (1) the amount of time involved, with some students feeling that a 1hour block would be sufficient; and (2) the use of videotape simulations rather than real patient encounters (regrettable but unavoidable for this type of presentation).

\section{Feedback from faculty}

Each year, course faculty met after the quarter to discuss program results, to decide whether to continue this component of the curriculum, and to plan for more effective teaching in the coming year.
While minor revisions were made each year, the faculty continue to conclude that the program is a good use of their time and worthy of continuation.

\section{Conclusions and discussion}

We have successfully implemented the McDaniel family program in small groups early in our curriculum the past three years. The program provides a vehicle for interaction between students and family physicians, which students find particularly valuable in the midst of their basic science training. Both students and faculty continue to evaluate the program favorably.

Our reseach suggests that the most significant impact of the program is on student attitudes toward modes of practice used in family and primary care medicine-team health care, ambulatory medicine, and an emphasis on cost containment. We are unable to report significant changes in either psychosocial attitudes or in future career choice; however, we believe that the change in attitudes toward mode of practice is by itself an accomplishment.

We plan to continue the McDaniel family program in our curriculum, and we are working on further developments in the areas of implementation and research. This year, for example, one of the group leaders exposed his students to real patients at the outlying clinic where he practices. This innovation was strongly supported by the students, and it is one we may seek to expand. In addition, we are considering ways to encourage students to take an even more active role in the small groups, perhaps by implementing some of the techniques used in the McMaster curriculum. ${ }^{5}$

In the area of research, we are revising and expanding the attitude survey to incorporate additional components of attitudes toward family medicine. We also plan to extend administration of the survey over the remainder of the curriculum; this may allow us to detect long-term changes in attitudes and career choice.

In conclusion, this program helps to underscore our college's commitment to family medicine. It seems to present family physicians and their role in health care in a positive way.

1. Parkerson, G.R., et al.: Medical care for the McDaniel family. Study guide (revised edition), workshop sessions 1-6; instructor's manual (revised edition). Duke-Watts Family Medicine Program, Duke University Medical Center. Health Sciences Consortium, Inc., Carrboro, North Carolina, 1983

2. Parkerson, G.R., et al.: Communications. A compact course in family medicine for preclinical medical students. J Med Educ 55:872-4, Oct 80 
3. Kim, J., and Mueller, C.W.: Factor analysis. Statistical methods and practical issues. Quantitative applications in the social sciences, no. 14 Sage Publications, Beverly Hills, California, 1978

4. Statistical analysis system. Ed. 5. SAS Institute, Inc., Cary, North Carolina, 1985

5. Neufeld, V.R., and Barrows, H.S.: The McMaster philosophy. An approach to medical education. J Med Educ 49:1040-50, Nov 74

Bjorn, J.C., and Cross, H.D.: Problem-oriented practice. McGraw-Hill, New York, 1970

Hurst, J.W., and Walker, H.K. The problem-oriented system. Medcom, New York, 1972

Weed, L.L.: Medical records, medical evaluation, and patient care: The problem-oriented record as a basic tool. Case Western Reserve University Press, Cleveland, Ohio, 1969

From Ohio University College of Osteopathic Medicine, Athens, Ohio, where Dr. Raub is an assistant professor of family medicine, and course coordinator, Osteopathic Clinical Practice II; Dr Baker is assistant dean, Educational Development and Resources; and Ms. Mann is coordinator of faculty development.

Dr. Raub, Box 714, 2 Kent Drive, Athens, Ohio 45701 